Pacific Journal of Mathematics

SOME ISOPERIMETRIC INEQUALITIES FOR THE 


\title{
SOME ISOPERIMETRIC INEQUALITIES FOR THE EIGENVALUES OF VIBRATING STRINGS
}

\author{
DAVID C. BARNES
}

If a string with integrable density function $p(x)$ is fixed at the points $x=0, x=a$ then the natural frequencies of vibration are determined by the eigenvalues of the SturmLiouville System

$$
y^{\prime \prime}+\lambda p(x) y=0 \quad y(0)=y(a)=0 .
$$

These eigenvalues depend on the density function $p(x)$ and we denote them accordingly by $\lambda_{n}(p)$,

$$
0<\lambda_{1}(p)<\lambda_{2}(p)<\cdots \text {. }
$$

In this work we investigate the nature of the density functions which yield the largest and smallest possible value for $\lambda_{n}(p)$ assuming that the average value of the density $p(x)$ defined by

$$
P(x)=\frac{1}{x} \int_{0}^{x} p(\zeta) d \zeta
$$

is restricted in some manner.

We assume for example that $P(x)$ is decreasing or that $P(x)$ is concave (see Theorems 4 and 7 below).

Assuming a string of given mass $m$ and a bounded density function $p(x), 0 \leqq p(x) \leqq H$, M. G. Krein [8] has obtained the sharp bounds

$$
\frac{4 H n^{2}}{m^{2}} X\left(\frac{m}{a H}\right) \leqq \lambda_{n}(p) \leqq \frac{\pi^{2} n^{2} H}{m^{2}},
$$

where $X(t)$ is the smallest positive root of the equation

$$
\sqrt{\bar{X}} \tan \sqrt{\bar{X}}=\frac{t}{1-t}
$$

Banks [1], [2], [5] has obtained some improvements of the Krein inequality by imposing various restrictions on the density function $p(x)$. Schwarz [12], Nehari [10], [11], Banks [4] and Maki [9] have obtained additional related results.

Given numbers $m, H, a$ such that $m<a H$, and an integrable density functions $p(x)$ defined on $[0, a]$ for which

$$
0 \leqq p(x) \leqq H, \quad \int_{0}^{a} p(x) d x=m,
$$


then the function $p(x)$ will be said to be of class

$E_{1}(m, H, a)$ provided $P(x)$ is decreasing

$E_{2}(m, H, a)$ provided $P(x)$ is increasing

$E_{3}(m, H, a)$ provided $P(x)$ is convex

$E_{4}(m, H, a)$ provided $P(x)$ is concave.

These function classes are related to certain classes studied by Bruckner and Ostrow [6] defined as follows:

A function $p(x)$ which satisfies (2) and

$$
\lim _{x \rightarrow 0+} p(x)=p(0)=0
$$

will be said to be of class

$K_{1}(m, H, a)$ provided $p(x)$ is convex,

$K_{2}(m, H, a)$ provided $P(x)$ is convex,

$K_{3}(m, H, a)$ provided $p(x)$ is starshaped from above at the origin, that is

$$
p(\alpha x) \leqq \alpha p(x) \text { for all } x \in[0, a] \text { and for all } \alpha \in[0,1],
$$

$K_{4}(m, H, a)$ provided $p(x)$ is superadditive, that is for any $x, y \in[0, a]$ if $x+y \in[0, a]$ then $p(x+y) \geqq p(x)+p(y)$,

$K_{5}(m, H, a)$ provided $P(x)$ is starshaped from above at the origin, $K_{6}(m, H, a)$ provided $P(x)$ is superadditive.

It follows from the work of Bruckner and Ostrow that

$$
K_{i}(m, H, a) \subset K_{i+1}(m, H, a) \quad i=1,2, \cdots 5 .
$$

In [6] these class inclusions are shown to hold for continuous functions which vanish at the orgin. That is, if $K_{i}$ denotes the class of continuous functions contained in $K_{i}(m, H, a)$ then $K_{i} \subset K_{i+1}$. Thus the Baire class generated by $K_{i}$ is contained in the Baire class generated by $K_{i+1}$. Making use of the dominated convergence theorem it is easy to see that the classes $K_{i}(m, H, a)$ are closed under the operation of taking pointwise limits. Thus the inclusions (3) hold if $p(x)$ is a Baire function. This will be sufficient for our work since the functionals $\lambda_{n}(p)$ are not altered by changing $p(x)$ on a set of measure zero.

We now define corresponding classes of functions for the concave case. If the function $p(x)$ satisfies (2), it will be said to be of class, $J_{1}(m, H, a)$ provided $p(x)$ is concave $J_{2}(m, H, a)$ provided $P(x)$ is concave, $J_{3}(m, H, a)$ provided $p(x)$ is starshaped from below at the origin, that is $p(\alpha x) \geqq \alpha p(x)$ for all $x \in[0, a]$ and for all $\alpha \in[0,1]$,

$J_{4}(m, H$, a) provided $p(x)$ is subadditive, for any $x, y \in[0, a]$ if $x+y \in[0, a]$ then $p(x+y) \leqq p(x)+p(y)$,

$J_{5}(m, H, a)$ provided $P(x)$ is starshaped from below at the origin, $J_{6}(m, H, a)$ provided $P(x)$ is subadditive. 
Note that in this case we do not assume that $p(0)=0$.

In Theorems 1, 2, and 3 below we will give a general method which allows one to calculate the extremal values of $\lambda_{n}(p)$ whenever $p(x)$ belongs to one of the above function classes. In some cases these calculations may be carried through to completion and explicit numerical bounds are given. In other cases however only general information concerning the extremal function for $\lambda_{n}(p)$ is given.

We review briefly the results we shall need from the SturmLiouville theory of differential equations (see [7]). Given the system (1), then corresponding to each eigenvalue $\lambda_{n}(p)$ there is an eigenfunction $u_{n}$ which is uniquely determined except for a multiplicative factor. It has exactly $n+1$ zeros in the interval $[0, a]$ which we denote by $x_{i}$,

$$
0=x_{0}<x_{1}<\cdots<x_{n}=a, \quad u_{n}\left(x_{i}\right)=0 .
$$

We may assume $u_{n}(x) \geqq 0$ in $\left[0, x_{1}\right]$. It then follows from (1) that $u_{n}$ is concave in $\left[0, x_{1}\right]$, convex in $\left.] x_{1}, x_{2}\right]$ etc. Thus in each of the intervals $\left[x_{i}, x_{i+1}\right], u_{n}^{2}(x)$ will have a unique maximum value which will be attained for some point $\alpha_{i}$,

$$
\alpha_{i} \in\left(x_{i}, x_{i+1}\right), \quad u_{n}^{2}\left(\alpha_{i}\right)=\max _{x_{i} \leqq x \leqq x_{i+1}} u_{n}^{2}(x) .
$$

The point $\alpha_{i}$ may or may not be uniquely determined. It follows that

$$
u_{n}(x) u_{n}^{\prime}(x) \geqq 0 \quad \text { if } \quad x \in\left[x_{i-1}, \alpha_{i}\right]
$$

and

$$
u_{n}(x) u_{n}^{\prime}(x) \leqq 0 \quad \text { if } \quad x \in\left[\alpha_{i}, x_{i}\right]
$$

In order to investigate the lower bounds on $\lambda_{n}(p)$ we make use of the following theorem (compare [11] and [3].)

THEOREM 1. Let $p(x)$ and $q(x)$ be two density functions. Let $u_{n}$ be the nth eigenfunction of (1) corresponding to the eigenvalue $\lambda_{n}(p)$. If

$$
u_{n} u_{n}^{\prime} \int_{0}^{x}[p(\zeta)-q(\zeta)] d \zeta \geqq 0 \quad \text { for all } x \in[0, a]
$$

then

$$
\lambda_{n}(q) \leqq \lambda_{n}(p)
$$

Proof. Let $x_{i}$ be the nodal points of the string with density $p(x)$ (see (4)). An integration by parts and (6) implies 


$$
0 \leqq \int_{x_{i-1}}^{x_{i}} 2 u_{n} u_{n}^{\prime} x[P(x)-Q(x)] d x=-\int_{x_{i-1}}^{x_{i}} u_{n}^{2}[p(x)-q(x)] d x .
$$

Therefore

$$
\int_{x_{k-1}}^{x_{k}} u_{n}^{2} p(x) d x \leqq \int_{x_{i-1}}^{x_{i}} u_{n}^{2} q(x) d x .
$$

Following Banks [1] we fix the string at its nodal points $x_{i}$. It is known that [7]

$$
\lambda_{n}(p)=\frac{\int_{x_{i-1}}^{x_{i}} u_{n}^{\prime 2} d x}{\int_{x_{i-1}}^{x_{i}} u_{n}^{2} p(x) d x} \quad i=1,2, \cdots n .
$$

by (7) we have

$$
\lambda_{n}(p) \geqq \frac{\int_{x_{i-1}}^{x_{i}} u_{n}^{\prime 2} d x}{\int_{x_{i-1}}^{x_{i}} u_{n}^{2} q(x) d x} \geqq \inf _{y \in C^{\prime}} \frac{\int_{x_{i-1}}^{x_{i}} y^{\prime 2} d x}{\int_{x_{i-1}}^{x_{i}} y^{2} q(x) d x}
$$

where $y\left(x_{i}\right)=0 i=0,1,2, \cdots n$. It follows that

$$
\lambda_{n}(p) \geqq \max _{1 \leqq i \leqq n} \inf _{y \in C^{\prime}} \frac{\int_{x_{i-1}}^{x_{i}} y^{\prime 2} d x}{\int_{x_{i-1}}^{x_{i}} y^{2} q(x) d x} .
$$

But the quantity on the right is greater than the $n$th eigenvalue of a string of density $q(x)$ [7] whence $\lambda_{n}(p) \geqq \lambda_{n}(q)$.

The upper bounds on the functionals $\lambda_{n}(p)$ are more difficult to handle. We shall use methods from the calculus of variations. In order to use these methods we must know that the functional $\lambda_{n}(p)$ actually attains its last upper bound.

THEOREM 2. Let $E$ be any one of the function classes $E_{i}(m, H, a)$ for $i=1,2,3,4, K_{i}(m, H, a)$ for $i=1,2,3,5,6$ or $J_{i}(m, H, a)$ for $i=1,2,3,5,6$. Let $\lambda_{n}(p)$ be the nth eigenvalue of (1). Then there is a function $\rho(x) \in E$ such that

$$
\max _{p \in E} \lambda_{n}(p)=\lambda_{n}(p), \quad \rho(x) \in E .
$$

The proof uses a result of Krein [8] which may be stated as follows: Let $M$ be the set of all measurable functions on $[0, a]$ such that 


$$
0 \leqq p(x) \leqq H, \quad \int_{0}^{a} p(x) d x=m .
$$

Then there is a function $q(x) \in M$ such that

$$
\int_{0}^{x} p_{k}(\zeta) d \zeta \rightarrow \int_{0}^{x} q(\zeta) d \zeta \quad \text { as } K \rightarrow \infty
$$

The convergence is uniform in $x$ and

$$
\lambda_{n}\left(p_{k}\right) \rightarrow \lambda_{n}(q)=\max _{p \in M} \lambda_{n}(p) .
$$

Krein's proof may be modified simply by selecting $p_{k}(x) \in E$ (note that $E \subseteq M)$. Thus it is only necessary to show that $q(x) \in E$ provided $p_{k}(x) \in E$.

Suppose for example that $p_{k}(x) \in E_{4}(m, H, a)$ so that $p_{k}(x)$ is concave on the average. Thus the corresponding sequence of average values $\left\{P_{k}(x)\right\}$ is a sequence of concave functions which converge uniformly to

$$
Q(x)=\frac{1}{x} \int_{0}^{x} q(\zeta) d \zeta
$$

in any interval of the form $[\varepsilon, a] . \varepsilon>0$. Thus $Q(x)$ is concave and $q(x) \in E_{4}(m, H, a)$. The proof of the other cases $p_{k}(x) \in E_{i}(m, H, a)$ $i=1,2,3$ follows in a like manner. We must consider in more detail those function classes which are not defined in terms of an integral relationship. Suppose for example that $p_{k}(x) \in J_{3}(m, H, a)$ so that $p_{k}(\alpha x) \geqq \alpha p_{k}(x)$. It follows that

$$
\frac{1}{x-y} \int_{y}^{x} p_{k}(\alpha \zeta) d \zeta \leqq \frac{\alpha}{x-y} \int_{y}^{x} p_{k}(\zeta) d \zeta \quad x \neq y .
$$

If we first let $k \rightarrow \infty$ and then let $y \rightarrow x$ in the above inequality we find $q(\alpha x) \geqq \alpha q(x)$ for almost all $x$. Clearly we may redefine $q(x)$ on a set of measure zero so that this inequality holds for all $x$. With this new definition of $q(x)$ it follows that $q(x) \in J_{3}(m, H, a)$. It is now easy to complete the proof of Theorem 2 which we leave to the reader.

It is known [2] that the first variation of the functional $\lambda_{n}(p)$ subject to the condition

$$
\int_{0}^{a} p(x) d x=m
$$

is

$$
\delta \lambda_{n}(p)=-\lambda_{n}(p) \int_{0}^{a} u_{n}^{2} \delta p(x) d x,
$$

where $u_{n}$ is the $n$th normalized eigenfunction corresponding to $\lambda_{n}(p)$ and 


$$
\int_{0}^{a} \delta p(x) d x=0
$$

The following theorem will be used to obtain information about the upper bounds on $\lambda_{n}(p)$.

Theorem 3. Let $E$ be one of the function classes $E_{i}(m, H, a)$ $i=1,2,3,4, K_{i}(m, H, a) i=1,2,3,5,6$ or $J_{i}(m, H, a)$ for $i=1,2,3$, 5,6. Let $\lambda_{n}(p)$ be the nth eigenvalue of (1) and let $u_{n}$ be the corresponding normalized eigenfunction. Suppose that $\rho(x)$ is the maximizing function for $\lambda_{n}(p)$,

$$
\max _{p \in E} \lambda_{n}(p)=\lambda_{n}(\rho) \text {. }
$$

Suppose also that a mapping of $E$ into $E$ is given by $p(x) \rightarrow \bar{p}(x)$ which satisfies

$$
u_{n} u_{n}^{\prime} \int_{0}^{x}[p(\zeta)-\bar{p}(\zeta)] d \zeta \leqq 0 \quad \text { for } x \in[0, a] .
$$

Then $\rho(x)$, the maximizing function of $\lambda_{n}(p)$, is a fixed point of the mapping

$$
p(x)=\bar{\rho}(x)
$$

Proof. If $\rho(x)$ is the maximizing function for $\lambda_{n}(p)$ then there exists some $\bar{\rho}(x)$ such that

$$
u_{n} u_{n}^{\prime} \int_{0}^{x}[\rho(\zeta)-\bar{\rho}(\zeta)] d \zeta \leqq 0
$$

A simple integration by parts yields

$$
-\int_{0}^{a} u_{n}^{2}[\rho(x)-\bar{\rho}(x)] d x \leqq 0 .
$$

We now take a variation in $\rho(x)$ given by $\delta \rho(x)=\varepsilon[\bar{\rho}(x)-\rho(x)]$, $0<\varepsilon<1$. We note that $\rho(x)+\delta \rho(x) \in E$. But now

$$
\delta \lambda_{n}(\rho)=\lambda_{n}(\rho) \int_{0}^{a} u_{n}^{2} \delta \rho(x) d x \geqq 0 .
$$

However $\rho(x)$ is the maximizing function for $\lambda_{n}(p)$ and thus $\delta \lambda_{n}(\rho) \leqq 0$. Therefore $\delta \lambda_{n}(\rho)=0$. This together with (8) and (9) yields

$$
u_{n} u_{n}^{\prime} \int_{0}^{x}[\rho(\zeta)-\bar{\rho}(\zeta)] d \zeta=0 \quad \text { for } x \in[0 a]
$$

Since $u_{n}$ has only $n+1$ simple zeros we obtain 


$$
u_{n}^{\prime} \int_{0}^{x}[\rho(\zeta)-\bar{\rho}(\zeta)] d \zeta=0
$$

We cannot divide by $u_{n}^{\prime}$ since there may exist a set of positive measure on which $u_{n}^{\prime}=0$; and indeed such eigenfunctions will play an important role in the proof of Theorem 4. Let $A$ be the set of points is $[0, a]$ on which $u_{n}^{\prime}(x)=0 . \quad A$ is a closed set. Since $u_{n}^{\prime \prime}=-\lambda_{n}(\rho) \rho(x) u_{n}, u_{n}$ is locally concave or convex depending on its sign. Therefore $A$ must consist of a finite number of closed intervals, some of which may be only a single point. The complement of $A, A^{c}$ must therefore be open and from (10) it follows that $\rho(x)=\bar{\rho}(x)$ for almost all $x \in A^{c}$. Suppose now that $x \in A^{0}$, the interior of $A$. Thus $u_{n}^{\prime \prime}(x)=0$ and from (1) it follows that $\rho(x)=0$ on $A^{0}$. It follows that

$$
\int_{0}^{a} \rho(x) d x=\int_{A^{c}} \rho(x) d x=\int_{A^{c}} \bar{\rho}(x) d x=\int_{0}^{a} \bar{\rho}(x) d x,
$$

where we have used the fact that $\rho(x)$ and $\bar{\rho}(x)$ must have the same integral over $[0, a]$. We obtain

$$
\int_{A^{0}} \bar{\rho}(x) d x=0
$$

and therefore $\bar{\rho}(x)=0$ for almost all $x \in A^{0}$. Thus $\rho(x)=\bar{\rho}(x)$ almost everywhere which completes the proof of Theorem 3.

Finally we note that we may consider the eigenvalue problem

$$
u^{\prime \prime}+u \rho(t) u=0 \quad u(0)=u(1)=0
$$

where $\rho(t)=\{a p(a t) / m\}, 0 \leqq t \leqq 1$ instead of (1). Denoting the eigenvalues of (11) by $\mu_{n}(\rho)$ we see that

$$
\mu_{n}(\rho)=\operatorname{ma\lambda }_{n}(p) \text { and } \int_{0}^{1} \rho(t) d t=1 .
$$

Since all the conditions on $p(x)$ which we study here will also be satisfied by $\rho(t)$ we see that no loss of generality is involved by considering a string of unit length and unit mass. The relationship between the eigenvalues is given by (12).

2. Bounds on $\lambda_{n}(p)$ in case $p(x) \in E_{i}(m, H, a)$. As an example of the preceding ideas we will obtain a sharp upper bound on $\lambda_{n}(p)$ whenever $p(x)$ is decreasing on the average, $p(x) \in E_{1}(m, H, a)$.

THEOREM 4. Let $\lambda_{n}(p)$ be the nth eigenvalue of a vibrating string with fixed end points and a density function $p(x)$ which is decreasing on the average. If $p(x) \in E_{1}(m, H, a)$ then 


$$
\lambda_{n}(p) \leqq \frac{\pi^{2} n^{2} H}{m^{2}}\left[\frac{2 n-1+\sqrt{m / a H}}{2 n}\right]^{2}
$$

The inequality is sharp and equality is attained uniquely for a string of density $\rho(x) \in E_{1}(m, H, a)$ given by

$$
\rho(x)=\left\{\begin{array}{cl}
H & 0 \leqq x \leqq s \\
0 & s<x<t \\
m / a & t \leqq x \leqq a
\end{array}\right.
$$

where

$$
s=\frac{(2 n-1) m a}{(2 n-1) H a+\sqrt{m H a}}, \quad t=\frac{(2 n-1) a^{2} H}{(2 n-1) a H+\sqrt{m H a}} .
$$

REMARK. We note that Krein's upper bound on $\lambda_{n}(p)$ (assuming only that $0 \leqq p(x) \leqq H$ and $\int_{0}^{a} p(x) d x=m$ ) is given by $\pi^{2} n^{2} H / m^{2}$. Thus if we assume in addition that $p(x)$ is decreasing on the average we are able to improve this result by the factor indicated in the theorem.

Proof of Theorem 4. Let $p(x) \in E_{1}(m, H, a)$ and let $u_{n}$ be the $n$th normalized eigenfunction corresponding to $\lambda_{n}(p)$. Let $x_{i}$ be the nodes of the string with density $p(x)$ and let $\alpha_{i}$ be the sequence of maximizing points for $u_{n}^{2}$ as in (4) and (5). Define constants $m_{i}$ and $M_{i}$ by

$$
m_{i}=\int_{0}^{\alpha_{i}} p(x) d x, \quad M_{i}=\int_{0}^{x_{i}} p(x) d x, \quad m_{0}=M_{0}=0 .
$$

Furthermore define constants $k_{i}, s_{i}, t_{i}$ by

$$
k_{0}=H, k_{i}=\frac{m_{i}}{x_{i}}, \quad \mathrm{~s}_{1}=\frac{m_{1}}{H}, \quad s_{i}=x_{i-1} \frac{m_{i}}{M_{i-1}}, \quad t_{i}=x_{i} \frac{m_{i}}{M_{i}} .
$$

We now show that

$$
x_{i-1} \leqq s_{i} \leqq \alpha_{i} \leqq t_{i} x_{i} .
$$

We know that $x_{i-1}<\alpha_{i}<x_{i}$. Thus

$$
M_{i-1} \leqq m_{i} \leqq M_{i} \quad \text { and } \quad \frac{M_{i-1}}{x_{i-1}} \geqq \frac{m_{i}}{\alpha_{i}} \geqq \frac{M_{i}}{x_{i}} .
$$

The first set of inequalities above implies that $x_{i-1} \leqq s_{i}$ and $t_{i} \leqq x_{i}$. The second set of inequalities implies that $s_{i} \leqq \alpha_{i} \leqq t_{i}$. Therefore (13) holds.

We now define functions $\bar{p}(x), \bar{f}(x)$ and $f(x)$ by 


$$
\begin{gathered}
\bar{p}(x)=\left\{\begin{array}{ll}
k_{i-1} & x_{i-1} \leqq x \leqq s_{i} \\
0 & \mathrm{~s}_{i}<x<t_{i} \\
k_{i} & t_{i} \leqq x \leqq x_{i}
\end{array} \quad, \quad \bar{f}(x)= \begin{cases}k_{i-1} x & x_{i-1} \leqq x \leqq s_{i} \\
m_{i} & s_{i} \leqq x \leqq t_{i} \\
k_{i} x & t_{i} \leqq x \leqq x_{i}\end{cases} \right. \\
f(x)=\int_{0}^{x} p(\zeta) d \zeta
\end{gathered}
$$

according to the definition of $s_{i}$ and $t_{i}$, the function $\bar{f}(x)$ is well defined, continuous and

$$
\bar{f}(x)=\int_{0}^{x} \bar{p}(\zeta) d \zeta
$$

It is easy enough to see that $\bar{f}(x) / x$ is decreasing and that $\bar{p}(x) \in E_{1}(m, H, a)$. This procedure defines a mapping of $E_{1}(m, H, a)$ into itself given by $p(x) \rightarrow \bar{p}(x)$. In order to apply Theorem 3 we shall show

$$
u_{n} u_{n}^{\prime}[f(x)-\bar{f}(x)] \leqq 0, \quad x \in[0, a] .
$$

Suppose first of all that $x \in\left[0, s_{1}\right]$. Now if there is some point $x_{0} \in\left[0, s_{1}\right]$ such that $f\left(x_{0}\right)>\bar{f}\left(x_{0}\right)$, then an easy generalization of the mean value theorem shows that $f^{\prime}(\zeta)=p(\zeta)>\bar{f}^{\prime}(\zeta)=H$ for some point $\zeta \in\left(0, s_{1}\right)$. This is a contradiction and we must have $f(x) \leqq \bar{f}(x)$, $x \in\left[0, s_{1}\right]$. We also know that $u_{n} u_{n}^{\prime} \geqq 0$ in $\left[0, s_{1}\right]$. Therefore (14) holds for $x \in\left[0, s_{1}\right]$. Now suppose $x \in\left[x_{i-1}, s_{i}\right], i=1,2, \cdots n$. In this case also we have $u_{n} u_{n}^{\prime} \geqq 0$. Since $p(x)$ is decreasing on the average we have

$$
\frac{m_{i-1}}{x_{i-1}} \geqq \frac{1}{x} \int_{0}^{x} p(\zeta) d \zeta
$$

Therefore $\bar{f}(x) \geqq f(x)$ and (14) follows. Suppose now that $x \in\left[s_{i}, \alpha_{i}\right]$. Since $x \leqq \alpha_{i}$ it follows that

$$
f(x)=\int_{0}^{x} p(\zeta) d \zeta \leqq \int_{0}^{\alpha_{i}} p(\zeta) d \zeta=m_{i}=\bar{f}(x)
$$

Since $u_{n} u_{n}^{\prime} \geqq 0$ we obtain (14). We now suppose $x \in\left[\alpha_{i}, t_{i}\right]$. An argument similar to that used in (16) shown $\bar{f}(x) \leqq f(x)$. Since however $u_{n} u_{n}^{\prime} \leqq 0$ in this case we obtain (14). Finally, suppose $x \in\left[t_{i}, x_{i}\right]$. In this case an argument similar to that used in (15) will show that $f(x) \geqq \bar{f}(x)$. Since $u_{n} u_{n}^{\prime} \leqq 0$ in this interval, (14) will hold.

Thus a mapping $p(x) \rightarrow \bar{p}(x)$ of $E$ into $E$ is defined which satisfies the conditions of Theorem 3. Therefore, if $\rho(x)$ is the maximizing function for $\lambda_{n}(p)$ over $E_{1}(m, H, a)$ then $\rho(x)=\bar{\rho}(x)$. In order to simplify the notation, we assume that the original function $p(x)$ which we started with is the maximizing function, that is $p(x)=\rho(x)=\bar{\rho}(x)$. 
Note that $u_{n}$ is now the $n$th eigenfunction corresponding to $\lambda_{n}(\rho)$.

Now we know $\rho(x)=\bar{\rho}(x)=0$ if $x \in\left(s_{i}, t_{i}\right)$ and $\alpha_{i}$, the maximizing point for $u_{n}^{2}$, is contained in $\left(s_{i}, t_{i}\right)$. Therefore $u_{n}$ is constant in the interval $\left(s_{i}, t_{i}\right)$ since $u_{n}$ satisfies

$$
u_{n}^{\prime \prime}+\lambda_{n}(\rho) \rho(x) u_{n}=0 \quad u_{n}(0)=u_{n}(a)=0 .
$$

We may now replace the above equation by the collection of equations

$$
\begin{aligned}
u_{n}^{\prime \prime}+\lambda_{i} u & =0, & u^{\prime}\left(s_{i+1}\right) & =u^{\prime}\left(t_{i}\right) x \in\left(s_{i}, t_{i}\right) \\
u^{\prime \prime}+\lambda H u & =0, & u(0) & =u^{\prime}\left(s_{1}\right)=0 \\
u^{\prime \prime}+\lambda \frac{m}{a} u & =0, & u^{\prime}\left(t_{n}\right) & =u(a)=0,
\end{aligned}
$$

where the 2nd eigenvalue of (18) and the first eigenvalue of (18) and the first eigenvalue of (19) and (20) are all equal to the $n$th eigenvalue of (17) (see [7]). Solving each of these equations in turn yields the equations for $\lambda$,

$$
\begin{aligned}
\sqrt{\lambda k_{i}}\left(x_{i}-t_{i}\right)=\frac{\pi}{2}, \quad i & =1,2, \cdots n-1 . \\
\sqrt{\lambda H s_{1}} & =\frac{T}{2} \\
\sqrt{\lambda \frac{m}{a}}\left(a-t_{n}\right) & =\frac{\pi}{2} .
\end{aligned}
$$

Making use of the fact that $\int_{0}^{a} \rho(x) d x=m$ we obtain

$$
s_{1} H+\sum_{i=1}^{n-1}\left(s_{i+1}-t_{i}\right) k_{i}+\left(a-t_{n}\right) \frac{m}{a}=m .
$$

Substituting for $s_{i}, t_{i}$ from equations (21) we easily obtain

$$
\lambda_{n}(p)=\frac{\pi}{2 m^{2}}\left[\sqrt{H}+\sum_{i=1}^{n-1} 2 \sqrt{k_{i}}+\sqrt{\frac{m}{a}}\right] .
$$

Therefore the largest possible value of $\lambda_{n}(\rho)$ will occur when the $k_{i}$ are as large as possible. We must therefore take $k_{i}=H i=1,2, \cdots n-1$. This yields the upper bound on $\lambda_{n}(p)$ given in Theorem 4. We now obtain the function $\rho(x)$. Obviously $\rho(x)$ must be of the form

$$
\rho(x)=\left\{\begin{array}{cc}
H & 0 \leqq x \leqq s \\
0 & s<x<t \\
\frac{m}{a} & t<x<a
\end{array}\right.
$$


for some choice of $s, t$ with $u_{n}^{\prime}(s)=u_{n}^{\prime}(t)=0$. Solving this system shows that the eigenvalue $\lambda$ must satisfy the equation

$$
\sqrt{\lambda H s}=\frac{\pi}{2}(2 n-1) \text {. }
$$

Solving this equation for $s$ and using the formula for $\lambda$ already given we obtain the required formula for $s$. The formula for $t$ is obtained in a similar fashion to complete the proof of Theorem 4.

We now consider the lower bound on $\lambda_{1}(p)$ whenever $p(x)$ is decreasing on the average. In this case it is not necessary to assume that $p(x)$ is bounded above in order to obtain useful lower bounds. We therefore set $H=+\infty$ and assume $p(x) \in E_{1}(m, \infty, a)$. In case $p(x)$ is not only decreasing on the average but is actually decreasing, Banks [1] has given a sharp lower bound on $\lambda_{1}(p)$. The following theorem is a generalization of his result.

THEOREM 5. Let $\lambda_{1}(p)$ be the first eigenvalue of a vibrating string having fixed end points and a density function $p(x)$ which is decreasing on the average. If the total mass is $m$ and the length of the string is $a$, so that $p(x) \in E_{1}(m, \infty, a)$ then

$$
\operatorname{ma} \lambda_{1}(p) \geqq \lambda_{0}
$$

where $\lambda_{0}=7.88 \ldots$. The inequality is sharp and equality is attained for a string of density $q(x)$ given by

$$
q(x)=\left\{\begin{array}{cc}
\frac{m}{a t_{0}} & 0 \leqq x \leqq a t_{0} \\
0 & a t_{0} \leqq x \leqq a
\end{array}\right.
$$

where $t_{0}=.643 \cdots$.

Proof. In view of (12) we may assume that $m=a=1$. The general case will follow immediately. Now let $p(x) \in E_{1}(1, \infty, 1)$ and let $u$ be the eigenfunction of (1) corresponding to $\lambda_{1}(p)$. Let $\alpha$ be the maximizing point for $u^{2}(x), x \in[0,1]$ and define constants $H_{1}, m_{1}$ by

$$
m_{1}=\int_{0}^{\alpha} p(\zeta) d \zeta, \quad H_{1}=\frac{m_{1}}{\alpha} .
$$

Now define a density function $q_{t}(x)$ by

$$
q_{t}(x)=\left\{\begin{array}{ll}
H_{1} & 0 \leqq x \leqq t \\
0 & t \leqq x \leqq 1,
\end{array} \quad t=\frac{1}{H_{1}} .\right.
$$

Note that $q_{t}(x) \in E_{1}(1, \infty 1)$. We may assume that the eigenfunction 
$u$ is positive in $(0,1)$. We shall show

$$
u^{\prime} \int_{0}^{x}\left[p(\zeta)-q_{t}(\zeta)\right] d \zeta \leqq 0, \quad x \in[0,1]
$$

Define functions $f(x), g(x)$ by

$$
g(x)=\int_{0}^{x} q_{t}(\zeta) d \zeta \quad f(x)=\int_{0}^{x} p(\zeta) d \zeta \quad x \in[0,1] .
$$

Since $p(x)$ is decreasing on the average it follows that $f(x)$ is starshaped from below, that is $f(\alpha x) \geqq \alpha f(\mathrm{x})$ for all $x, \alpha \in[0,1]$ (see [6] Lemma 3). Now $f(\alpha)=g(\alpha)$ and $f(1)=g(1)$. From this it follows that

$$
\begin{array}{ll}
\int_{0}^{x} p(\zeta) d \zeta \geqq \int_{0}^{x} q_{t}(\zeta) d \zeta & x \in[0, \alpha] \\
\int_{0}^{x} p(\zeta) d \zeta \leqq \int_{0}^{x} q_{t}(\zeta) d \zeta & x \in[\alpha, 1] .
\end{array}
$$

Taking account of the sign of $u^{\prime}$ we see that (22) holds. We may now apply Theorem 1 to obtain $\lambda_{1}(q t) \leqq \lambda_{1}(p)$. Now the eigenvalue $\lambda_{1}\left(q_{t}\right)$ is a function of $t$. Banks [1] Theorem 2.1 has calculated the minimum value of this function. We may apply his results to complete the proof of Theorem 5 .

Now Theorem 5 deals only with the first eigenvalue $\lambda_{1}(p)$. In general it seems to be very difficult to obtain a precise lower bound on $\lambda_{n}(p)$. One can however pin the string down at its nodal points and consider it to be made up of $n$ separate parts. The $n$th eigenvalue $\lambda_{n}(p)$ will then be equal to the first eigenvalue of each separate part (see [7]). If one then applies a construction similar to that used in Theorem 5 to the $n$ parts of the string one obtains.

THEOREM 6. Let $\lambda_{n}(p)$ be the nth eigenvalue of a vibrating string with fixed end points and density function $p(x)$ which is decreasing on the average. If the total mass is $m$ so that $p(x) \in E_{1}(m, \infty, a)$ then there is a density function $q(x) \in E_{1}(m, \infty, a)$ such that

$$
\lambda_{n}(q) \leqq \lambda_{n}(p) \text {. }
$$

Here $q(x)$ has the form

$$
q(x)=\left\{\begin{array}{ll}
0 & x_{k-1} \leqq x \leqq s_{k} \\
H_{k} & s_{k} \leqq x \leqq t_{k} \\
0 & t_{k}<x \leqq x_{k}
\end{array} \quad k=1,2, \cdots n\right.
$$

The points $x_{k}$ are the zeros of the eigenfunction $u_{n}$ corresponding to the density $p(x)$. The constants $H_{k}, s_{k}, t_{k}$ satisfy 


$$
\begin{gathered}
s_{1}=0, \quad H_{k+1} s_{k+1}=H_{k} t_{k}, \\
H=H_{1} \geqq H_{2} \geqq \cdots \geqq H_{n}, \quad \sum_{k=1}^{n} H_{k}\left(t_{k}-s_{k}\right)=m .
\end{gathered}
$$

Banks ([2], Th. 4) has shown that if $p(x)$ is concave then $\lambda_{1}(p) \leqq$ $\pi^{2} / m a$ with equality in case $p(x)=m / a$ for all $x$. The following theorem generalizes this result.

THEOREM 7. Let $\lambda_{1}(p)$ be the first eigenvalue of a vibrating string with fixed end points and a density function $p(x)$ which is concave on the average. If the total mass is $m$ so that $p(x) \in E_{4}(m, H, a)$ for some constant $H$ then

$$
m a \lambda_{1}(p) \leqq \pi^{2}
$$

The inequality is sharp and equality is attained if

$$
p(x)=\frac{m}{a} \quad \text { for all } x \in[0,1] .
$$

Proof. Suppose $p(x) \in E_{4} m, H$, a) for some $H>2 m / a$. Let $u$ be the eigenfunction corresponding to $\lambda_{1}(p)$. If $\alpha$ is the maximizing point for $u^{2}$ we define $m_{1}, \bar{P}(x)$ and $\bar{p}(x)$ by

$$
m_{1}=\int_{0}^{\alpha} p(\zeta) d \zeta
$$

and

$$
\begin{aligned}
& \bar{P}(x)=\frac{m}{a}+(x-a) \frac{a m_{1}-\alpha m}{\alpha a(\alpha-a)} \\
& \bar{p}(x)=\frac{m}{a}+(2 x-a) \frac{a m_{1}-\alpha m}{\alpha a(\alpha-a)} .
\end{aligned}
$$

Obviously $\bar{P}(x)=1 / x \int_{0}^{x} \bar{p}(\zeta) d \zeta$. Thus $p(x) \in E_{4}(m, H, a)$. It follows from (23) that $\bar{P}(a)=P(a)=m / \alpha$ and $\bar{P}(\alpha)=P(\alpha)=m_{1} / \alpha$ (where, as usual, $P(x)$ is the average value of $p(x)$.) In view of the concavity of $P(x)$ it follows that

$$
\begin{array}{ll}
P(x) \leqq \bar{P}(x) & \text { for } x \in[0, \alpha] \\
P(x) \leqq \bar{P}(x) & \text { for } x \in[\alpha,) a] .
\end{array}
$$

Thus we obtain

$$
u u^{\prime}[P(x)-\bar{P}(x)] \leqq 0 \quad \text { for } x \in[0, a] .
$$

Therefore Theorem 3 implies that the maximum of $\lambda_{1}(p)$ will be attained for a linear function of the form $\bar{p}(x)$. We may now apply the result 
of Banks [2] to complete the proof of Theorem 7.

3. Bounds on $\lambda_{n}(p)$ in case $p(x) \in J_{i}(m, H, a)$. As a further example of the method we consider the minimum value of $\lambda_{1}(p)$ whenever $p(x) \in J_{5}(m, \infty, a)$ so that $p(x)$ is starshaped from below on the average. It turns out that the minimizing function for $J_{5}(m, \infty, a)$ actually belongs to $J_{3}(m, \infty, a)$. Since $J_{3}(m, \infty, a) \subseteq J_{4}(m, \infty, a) \subseteq$ $J_{5}(m, \infty a)$ (see [6]) it follows that $\lambda_{1}(p)$ has the same minimum value if $p(x)$ belongs to any one of these three classes.

THEOREM 8. Let $\lambda_{1}(p)$ be the first eigenvalue of a vibrating string having fixed end points and a density function $p(x)$ which is starshaped from below on the average. If the total mass is $m$ so that $p(x) \in J_{5}(m, \infty, a)$ then

$$
m a \lambda_{1}(p) \geqq \lambda_{0}
$$

where $\lambda_{0}=5.96 \cdots$. The inequality is sharp and equality is attained uniquely for a density function $q(x) \in J_{3}(m, \infty a)$ given by

$$
q(x)=\left\{\begin{array}{cc}
\frac{2 m}{t_{0}^{2} a^{2}} x & 0 \leqq x \leqq a t_{0} \\
0 & a t_{0}<x \leqq a
\end{array}\right.
$$

with $t_{0}=.590 \cdots$.

Proof. In view of (12) we may assume $m=a=1$. Now suppose $p(x) \in J_{5}(1, \infty, 1)$ and that $u$ is the first eigenfunction of (1) corresponding to the density $p(x)$. Let $\alpha$ be the maximizing point for $u^{2}$ and define

$$
m_{1}=\int_{0}^{\alpha} p(\zeta) d \zeta .
$$

Furthermore we define a function $Q(x)$ by

$$
Q(x)= \begin{cases}\frac{m_{1}}{\alpha^{2}} x & 0 \leqq x \leqq t \\ 1 / x & t \leqq x \leqq 1\end{cases}
$$

where $t$ is selected so that $Q(x)$ is continuous. Thus we require $m_{1} t^{2}=\alpha^{2}$. We note that $Q(x)$ is starshaped from below and that it is the average value of the function

$$
q_{t}(x)=\left\{\begin{array}{cc}
\frac{2 x}{t^{2}} & 0 \leqq x \leqq t \\
0 & t<x \leqq 1
\end{array}\right.
$$


Now $q_{t}(x)$ is also starshaped from below so that $q_{t}(x) \in J_{3}(1, \infty, 1)$. If $P(x)$ denotes the average value of $p(x)$ then in follows that $P(\alpha)=$ $Q(\alpha)$ and $P(1)=Q(1)$ (one can easily show that $\alpha \leqq t \leqq 1$.) Since $P(x)$ and $Q(x)$ are starshaped from below it follows that

$$
\begin{array}{ll}
P(x) \geqq Q(x) & x \in[0, \alpha] \\
P(x) \leqq Q(x) & x \in[\alpha, t] .
\end{array}
$$

In case $x \in[t, 1]$ we have

$$
P(x)=\frac{1}{x} \int_{0}^{x} p(\zeta) d \zeta \leqq \frac{1}{x} \int_{0}^{1} p(\zeta) d \zeta=1 / x=Q(x) .
$$

Taking account of the sign of $u u^{\prime}$, these three inequalities yield

$$
u u^{\prime}[P(x)-Q(x)] \geqq 0 \quad \text { for all } x \in[0,1] \text {. }
$$

We may now multiply this inequality by $x$ and apply Theorem 1 to obtain $\lambda_{1}\left(q_{t}\right) \leqq \lambda_{1}(p)$, Now $\lambda_{1}\left(q_{t}\right)$ is a function of the number $t \in[0,1]$. In order to complete the proof of Theorem 7 we must calculate its minimum value.

Since $q(x)=0$ for $x \in[t, l]$ it follows that $\lambda_{1}(q)$ is the first eigenvalue of the system

$$
u^{\prime \prime}+\lambda \frac{2 x}{t^{2}} u=0, u(0)=0, u(t)+(1-t) u^{\prime}(t)=0 .
$$

In order to solve this equation we introduce the function $u^{*}(x)$ defined to be the solution of

$$
u^{\prime \prime}+x u=0, \quad u(0)=0, \quad u^{\prime}(1)=1 .
$$

This function is tabulated in [13]. Now the first eigenfunction of (24) is

$$
u(x)=u^{*}\left(Z^{1 / 2} x\right), \quad Z=\left[2 \lambda / t^{2}\right]^{2 / 3}
$$

where $Z$ is the smallest positive root of the equation

$$
u^{*}\left(Z^{1 / 2} t\right)+Z^{1 / 2}(1-t) u^{*}\left(Z^{1 / 2} t\right)=0 .
$$

We define $\beta=Z^{1 / 2} t$ and $y(\beta)=u^{*}(\beta) / u^{* \prime}(\beta)$. Now (26) becomes

$$
u^{*}(\beta)+(B / t-\beta) u^{* \prime}(\beta)=0 .
$$

This equation together with the definition of $\beta$ and $y(\beta)$ may be used in conjunction with (25) to obtain

$$
2 \lambda=\frac{\beta^{3}}{t}=\beta^{2}[\beta-y(\beta)] .
$$


This equation defines $\lambda$ as a function of $\beta$. If we set $d \lambda / d \beta=0$ and simplify the resulting equation, making use of the relations

$$
u^{* \prime \prime}(\beta)=-\beta u^{*}(\beta) \text { and } \frac{d \lambda}{d \beta}=1+\beta y^{2}(\beta)
$$

we obtain

$$
\beta^{2} y^{2}(\beta)+2 y(\beta)-\beta=0 \quad \text { or } \quad y(\beta)+\frac{1+\left(2 \beta^{3}+1\right)^{1 / 2}}{\beta^{2}}=0
$$

(note that (27) and $t \leqq 1$ implies $y(\beta) \leqq 0$ ). We denote by $\beta_{0}=1.915 \cdots$ the smallest positive root of (28). This choice of $\beta$ will yield the smallest possible value of $\lambda$ which will be given by

$$
\lambda_{0}=1 / 2\left[1+B_{0}^{3}+\left(2 \beta_{0}^{3}+1\right)^{1 / 2}\right]=5.69 \cdots,
$$

This completes proof of Theorem 7. We note that for the higher eigenvalues $\lambda_{n}(p)$ the minimizing function for $J_{5}(m, \infty, a)$ will not belong to $J_{3}(m, \infty, a)$.

3. Some generalizations. There are obviously many other results concerning the size of $\lambda_{n}(p)$ which one may obtain using the method of Theorem 1, 2, and 3. Space does not permit inclusion of all of them but the basic ideas involved are the same as those in Theorems $4,5,6,7$, and 8 .

We now introduce a different type of average value function. We define the average value of a function $p(x)$ with respect to a function $r(x)$ by

$$
P(x)=\frac{1}{r(x)} \int_{0}^{x} p(\zeta) d \zeta, \quad P(0)=\lim _{x \rightarrow 0^{+}} P(x) .
$$

We may now define many different classes of density functions by placing some restriction on $P(x)$. For example we say that $p(x)$ is starshaped from below at the origin with respect to $x^{2}$ provided

$$
P(x)=\frac{1}{x^{2}} \int_{0}^{x} p(\zeta) d \zeta \quad \text { with } \quad P(\alpha x) \geqq \alpha P(x)
$$

for all $x \in[0, a], \quad \alpha \in[0,1]$.

As an example of the results which can be obtained along this line we give:

THeOREM 9. Let $\lambda_{1}(p)$ be the first eigenvalue of a vibrating string with fixed end points and a density function $p(x)$ which satisfies (29) above. If the total mass is $m$ then 


$$
m a \lambda_{1}(p) \geqq \lambda_{0}
$$

where $\lambda_{0}=5.33 \cdots$. The inequality is sharp and equality is attained for the function $q(x)$ given by

$$
q(x)= \begin{cases}3 x^{2} / t_{0}^{3} a^{3} & 0 \leqq x \leqq a t_{0} \\ 0 & a t_{0}<x \leqq a\end{cases}
$$

where $t_{0}=.566 \cdots$.

Proof. In view of (12) we may assume $m=a=1$. Suppose $p(x)$ is some function which satisfies (29) and let $u$ be the first eigenfunction of (1) corresponding to $\lambda_{1}(p)$. Let $\alpha$ be the maximizing point for $u^{2}$ and define $m_{1}=\int_{0}^{a} p(\zeta) d \zeta$. We now construct functions $Q(x)$ and $q_{t}(x)$ by

$$
Q(x)=\left\{\begin{array}{ll}
\frac{m_{1}}{\alpha^{3}} x & 0 \leqq x \leqq t \\
\frac{1}{x^{2}} & t \leqq x \leqq 1
\end{array} \quad q_{t}(x)= \begin{cases}3 x^{2} / t^{0} & 0 \leqq x \leqq t \\
0 & t<x \leqq 1,\end{cases}\right.
$$

where $t$ is selected so that $Q(x)$ is continuous. Thus we require $m_{1} t^{3}=\alpha^{3}$. Since $m_{1}<1$ we see $\alpha<t$. Thus $Q(\alpha)=m_{1} / \alpha^{2}=P(\alpha)$. Obviously $Q(1)=P(1)=1$. Applying (29) with $x=1$ we obtain $P(\alpha) \geqq$ $\alpha P(1)=\alpha$. Thus $m_{1} \geqq \alpha^{3}$ so $t \leqq 1$. Now it follows that

$$
Q(x)=1 / x^{2} \int_{0}^{x} q_{t}(\zeta) d \zeta
$$

and $Q(x)$ satisfies (29). We now show

$$
\begin{array}{ll}
P(x) \geqq Q(x) & x \in[0, \alpha] \\
P(x) \leqq Q(x) & x \in[\alpha, 1] .
\end{array}
$$

For $x \in[0, a]$ we have $P(x)=P(x / \alpha \alpha) \geqq(x / \alpha) P(\alpha)=Q(x)$ which is the first inequality. Now if $x \in[\alpha, t]$ then $P(\alpha)=P(x \alpha / x) \geqq(\alpha / x) P(x)$. This implies $P(x) \leqq Q(x)$. Finally we suppose $x \in[t, 1]$ so that

$$
\int_{0}^{x} p(\zeta) d \zeta \leqq 1
$$

Division by $x^{2}$ yields $P(x) \leqq Q(x)$ which proves (30). Taking account of the sign of $u u^{\prime}$ we obtain from (30)

$$
u u^{\prime}[P(x)-Q(x)] \geqq 0 \quad \text { for all } x \in[0,1] .
$$

We may now multiply this inequality by $x^{2}$ and apply Theorem 1 to obtain $\lambda_{1}(p) \geqq \lambda_{1}\left(q_{t}\right)$. Now $\lambda_{1}\left(q_{t}\right)$ is a function of the number $t \in[0,1]$. 
In order to complete the proof of Theorem 9 we must calculate its minimum value. We shall find it convenient to use functions $V_{1}(x), V_{2}(x)$ defined to be the solution of

$$
V^{\prime \prime}+x^{2} V=0 \quad V_{1}(0)=V_{2}^{\prime}(0)=1, \quad V_{1}^{\prime}(0)=V_{2}(0)=0 .
$$

These functions are tabulated in [13].

Now we note that $q_{t}(x)=0$ for $t \leqq x \leqq 1$. Thus $\lambda_{1}\left(q_{t}\right)$ is the first eigenvalue of the system

$$
V^{\prime \prime}+\lambda \frac{3 x^{2}}{t^{2}} V=0 \quad V(0)=0, \quad V(t)+\left(1-t V^{\prime}(t)=(t)=0 .\right.
$$

Solving this differential equation subject to $V(0)=0$ gives

$$
u(x)=V_{2}(\sqrt{Z} x) \quad \text { with } \quad \lambda=\frac{1}{3} Z^{2} t^{3} .
$$

Applying the second boundary condition defines $Z$ as a function of $t$ to be the smallest positive root of the equation

$$
V_{2}(\sqrt{Z} t)+(\sqrt{Z}-\sqrt{Z} t) V_{2}^{\prime}(\sqrt{Z} t)=0 .
$$

Define a number $\beta=\sqrt{Z} t$ and a function $y(\beta)=\left\{V_{2}(\beta) / V_{2}^{\prime}(\beta)\right\}$. Equation (32) becomes

$$
t=\frac{\beta}{\beta-y(\beta)} .
$$

Since $t \leqq 1$ we see $y(\beta) \leqq 0$. Thus $\lambda$ as a function of $\beta$ is given by

$$
3 \lambda=\beta^{4}-\beta^{3} y(\beta) \text {. }
$$

If we set $d \lambda / d \beta=0$ and simplify the resulting equation making use of the relation $d y / d \beta=1+\beta^{2} y^{2}$ we obtain

$$
\beta^{3} y^{2}(\beta)+3 y(\beta)-3 \beta=0
$$

or

$$
y(\beta)=\frac{V_{2}(\beta)}{V_{2}^{\prime}(\beta)}=-\frac{3+\sqrt{9+12 \beta^{4}}}{2 \beta^{3}} .
$$

This equation is the condition under which $d \lambda / d \beta=0$. Its smallest positive root is $\beta_{0}=1.733 \ldots$ (see [13]). Equations (33) and (34) give the corresponding values $\lambda_{0}=5.33 \cdots$ and $t_{0}=.566$. This completes the proof of Theorem 9 . 


\section{BIBLIOGRAPHY}

1. D. O. Banks, Bounds or the eigenvalues of some vibrating systems, Pacific J. Math. (2) 10 (1960), 439-474.

2. U Upper bounds for the eigenvalues of some vibrating systems, Pacific J. Math. (4) 11 (1961), 1183-1203.

3. — An integral inequality, Proc. Amer. Math. Soc. (5) 14 (1963), 823-828.

4. - Bounds for eigenvalues and generalized convexity, Pacific J. Math. (4) 12 (1963), 1031-1052.

5. - Lower bounds for the eigenvalues of the vibrating string whose density satisfies a Lipschitz condition, Pacific J. Math. (1) 20 (1967), 393-410.

6. A. M. Bruckner and E. Ostrow, Some function classes related to the class of convex function, Pacific J. Math. (4) 12 (1962), 1203-1215.

7. R. Courant, and D. Hilbert, Methods of mathematical physics, Interscience, New York, 1953.

8. M. Krein, On certain problems on the maximum and minimum of characteristic values and on Lyapunov zones of stability, Amer. Math. Soc. Trans. (2) 1 (1955), 163-187. 9. E. Makai, Über die Nullstellen von Funktionen die Lösungen Sturm-Liouville' scher Differentialgleichungen sind, Comm. Math. Helv. 16 (1943), 153-199.

10. Z. Nehari, Extremal problems for a class of functionals defined on convex sets, Bull. Amer. Math. Soc. 73 (1967), 584-591.

11. - Oscillation criteria for second order linear differential equations, Trans. Amer. Math. Soc. 85 (1957), 428-445.

12. B. Schwarz, On the extrema of the frequencies of nonhomogeneous strings with equimeasurable densities, J. Math. Mech. 10 (1961), 401-422.

13. A. Smirnov, Tables of Airy functions, Pergamon Press.

Received April 22, 1968. This work was based on the author's Doctoral Dissertation written under the direction of Professor D. O. Banks at the University of California at Davis.

WASHington State UNIVERSity

Pullman Washington 




\section{Pacific Journal of Mathematics \\ Vol. 29, No. 1 \\ May, 1969}

Jorge Alvarez de Araya, A Radon-Nikodým theorem for vector and operator

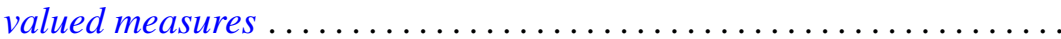

Deane Eugene Arganbright, The power-commutator structure of finite

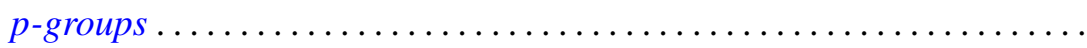

Richard Eugene Barlow, Albert W. Marshall and Frank Proschan, Some

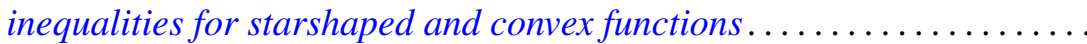

David Clarence Barnes, Some isoperimetric inequalities for the eigenvalues

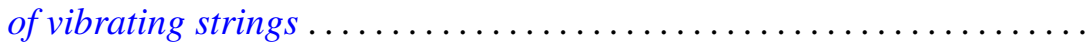

David Hilding Carlson, Critical points on rim-compact spaces ...........

Allan Matlock Weber Carstens, The lattice of pretopologies on an arbitrary

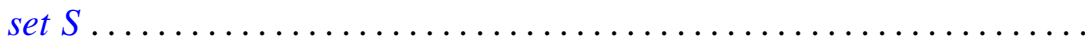

S. K. Chatterjea, A bilateral generating function for the ultraspherical

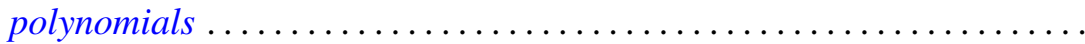

Ronald J. Ensey, Primary Abelian groups modulo finite groups ......... 77

Harley M. Flanders, Relations on minimal hypersurfaces ............ 83

Allen Roy Freedman, On asymptotic density in n-dimensions........... 95

Kent Ralph Fuller, On indecomposable injectives over artinian rings...... 115

George Isaac Glauberman, Normalizers of p-subgroups in finite groups . . . 137

William James Heinzer, On Krull overrings of an affine ring ........... 145

John McCormick Irwin and Takashi Ito, A quasi-decomposable abelian group without proper isomorphic quotient groups and proper isomorphic subgroups.

Allan Morton Krall, Boundary value problems with interior point boundary conditions

John S. Lowndes, Triple series equations involving Laguerre

polynomials

Philip Olin, Indefinability in the arithmetic isolic integers

Ki-Choul Oum, Bounds for the number of deficient values of entire functions whose zeros have angular densities..

R. D. Schafer, Standard algebras ....................

Wolfgang M. Schmidt, Irregularities of distribution. III.

Richard Alfred Tapia, An application of a Newton-like method to the Euler-Lagrange equation 